\title{
Community pharmacists perspectives about reasons behind antibiotics dispensing without prescription: a qualitative study.
}

\author{
Mansour A Mahmoud ${ }^{1 *}$, Mohammed Aldhaeefi ${ }^{2}$, Aziz Sheikh $^{3}$, Hisham Aljadhey $^{4}$ \\ ${ }^{1}$ Taibah University, Clinical and Hospital Pharmacy Department, College of Pharmacy, AL-Madinah Al-Munawara, \\ Saudi Arabia \\ ${ }^{2}$ King Saud Bin Abdulziz University for Health Sciences, College of Pharmacy, Riyadh, Saudi Arabia \\ ${ }^{3}$ The University of Edinburgh, the Usher Institute of Population Health Sciences and Informatics, Edinburgh, United \\ Kingdom \\ ${ }^{4}$ Saudi Food and Drug Authority, Riyadh, Saudi Arabia
}

\begin{abstract}
Background: Dispensing of antibiotics without prescription is a widespread practice particularly in developing countries. This may lead to bacterial resistance, extra cost and possible adverse drug events. Objective: To explore views, experiences and perceptions of community pharmacists about the reasons behind antibiotics dispensing without prescription.

Methods: This was a qualitative enquiry conducted among community pharmacists working in Riyadh city, Saudi Arabia. Interviews were conducted with the pharmacists using a semi structured interview guide. All interviews were audio-recorded, transcribed verbatim and then coded and analysed independently by two researchers.

Results: A total of 16 community pharmacists were interviewed. The majority of the pharmacists have dispensed antibiotics without prescription during their practice (14 out of 16). Indications for dispensing antibiotics included fever, sore throat, tonsillitis, urinary tract infections, common cold and cough. Factors contributing to the dispensing of antibiotics without prescriptions included, increasing demand by the public, financial considerations, promotion by pharmaceutical companies, the pharmacy management and time pressures. However, pharmacists mentioned that they did not dispense antibiotics without prescriptions for severe cases and patients with unknown or incomplete medical history.

Conclusion: Pharmacists reported several encouraging factors to dispense antibiotics without prescription. A combination of public education, professional training and enforcing regulations are likely to be important in ensuring patient safety and appropriate dispensing and use of antibiotics.
\end{abstract}

Keywords: Community pharmacists, Antibiotics, Dispensing, Qualitative study.

Accepted on November 23, 2018

\section{Introduction}

In Saudi Arabia, community pharmacists dispense prescription medications such as antibiotics without prescription and violate the low putting patients' lives in significant risk $[1,2]$. Antibiotics misuse is associated with several negative patient outcomes including bacterial resistance, increased cost and adverse drug events (ADEs) [3,4]. The recent report of the World Health Organization (WHO) reported that antibiotic resistance is very common all over the world and it is a serious public health problem [5]. Prevention of antibiotics resistance can be achieved by prohibiting dispensing of antibiotics without prescription, completion of the full prescription even if the patient feels better and avoiding the sharing of antibiotics with others [5]. It has been reported that in Saudi Arabia antibiotics were dispensed without prescription in $77.6 \%$ of community pharmacies in Riyadh city [1]. In Lebanon, a study reported that $32 \%$ of the antibiotics were dispensed without prescription [6]. A recent study conducted in the United Arab Emirates reported that $68.4 \%$ of antibiotics were sold without prescription [7]. Antibiotics dispensing without prescription is also common among African countries [8,9] and some European countries [10,11].

It has been several decades since the Saudi Ministry of Health has prohibited over the counter sale of antibiotics. However, these roles and regulations are often neglected by community pharmacists, the patients and pharmacy owners'. Although several studies in Saudi Arabia had assessed the prevalence of antibiotics dispensing without prescription, the reasons behind such practices remained unexplored. Knowing the reasons behind antibiotics dispensing without prescription could help 
healthcare authorities to design interventions that aim to improve community pharmacy practice. This study aimed to explore community pharmacists' views, experiences and perceptions about antibiotics dispensing without prescription in Riyadh, Saudi Arabia.

\section{Method}

\section{Study design}

This was a qualitative enquiry using a semi-structured interview [12] to explore the perspectives of community pharmacists about antibiotics dispensing without prescription.

\section{Study instrument}

An interview guide was developed after conducting an extensive literature review of similar studies. The interview guide was discussed among the authors and modified after few rounds of discussions. A pilot study was conducted with four community pharmacists to test the usefulness of the interview questions to address the study objectives and collect in-depth information about the study. The final topics included in the guide for interviews were: pharmacists perceptions about antibiotics dispensing without prescriptions and reasons for dispensing antibiotics without prescription. Quantitative data was collected on pharmacists demographic information and estimated number of antibiotics dispensed with and without prescriptions.

\section{Study sample}

Qualitative research usually uses non-probability sampling such as convenience sampling, purposive sampling, theoretical sampling, quota sampling and snowball sampling [13].

Snowball sampling was used to recruit the participants. Snowball sampling is one of the best sampling methods when the researcher is looking to interview subjects with certain characteristics. It involves identifying people with relevant characteristics and interviewing them and then these people are asked to suggest other people that have the same attributes as they do. The first community pharmacist was identified and each pharmacist was asked to suggest other pharmacists who may be interested to participate in the study. Interviews were continued until no new significant information was gathered from the transcripts.

\section{Interview process}

The investigator visited the community pharmacies once to conduct the interview. A written consent was obtained from each pharmacist upon the acceptance to participate in the study. All the interviews were conducted by a trained pharmacy student who is also one of the authors. Interviews were conducted with the pharmacists at their work place. Probing questions were used when necessary and pharmacists were given freedom to express their own views at the end of each interview. The time spent in each interview was approximately half an hour.
The ethical approval for this study was obtained from the Research and Ethics Committee at the School of Pharmacy, King Saud University.

\section{Data gathering and analysis}

All interviews' were audio-taped and transcribed verbatim into a computer and made available for analysis. The transcription was checked against the tape for accuracy. Then, common themes and sub-themes were generated from the transcripts by two independent researchers, using thematic content analysis. The themes were identified using inductive approach.

Thematic content analysis is one of the most commonly used methods to analyse qualitative data, and results in rich and detailed explanation of the data [14]. Saturation of data was reached after 16 interviews. Confidentiality and anonymity were maintained throughout the study and all community pharmacists were informed that no data that could lead to identification of any participant would be published in any form.

\section{Results}

Out of 22 community pharmacists approached a total of 16 community pharmacists were interviewed. All pharmacists were Male, non-Saudi nationals and the majority $(n=11$, $68.8 \%$ ) had five years or more of working experience (Table 1). The estimated number of antibiotics dispensed with and without prescriptions is summarized in Table 2. While exploring pharmacists' practices towards antibiotics dispensing, the majority of the community pharmacists said that they had dispensed antibiotics without prescription during their practice. Symptoms for which antibiotics were commonly dispensed included tonsillitis, sore throat, urinary tract infections, common cold and pharyngitis. In-depth analysis of the transcripts revealed two main themes. These were; factors perceived by pharmacists to be contributing to antibiotics dispensing without prescription and factors preventing pharmacists to dispense antibiotic without prescription.

Table 1. Demographic characteristics of the study population.

\begin{tabular}{lll}
\hline Community pharmacist & Age & Experience (y) \\
\hline 1 & 49 & 13 \\
\hline 2 & 46 & 17 \\
\hline 3 & 30 & 5 \\
\hline 4 & 29 & 5 \\
\hline 5 & 30 & 6 \\
\hline 6 & 36 & 13 \\
\hline 7 & 40 & 10 \\
\hline 8 & 32 & 8 \\
\hline 10 & 24 & 1 \\
\hline
\end{tabular}


study

\begin{tabular}{lll}
\hline 11 & 34 & 8 \\
\hline 12 & 36 & 6 \\
\hline 13 & 40 & 9 \\
\hline 14 & 41 & 2 \\
\hline 15 & 31 & 5 \\
\hline 16 & 26 & 1 \\
\hline
\end{tabular}

Table 2. Estimated number of antibiotics dispensed by pharmacist per day with and without prescription.

\begin{tabular}{|c|c|c|}
\hline \multirow[t]{2}{*}{ Community pharmacist } & \multicolumn{2}{|c|}{$\begin{array}{l}\text { Estimated number of antibiotics dispensed by } \\
\text { pharmacist per day }\end{array}$} \\
\hline & Without prescription & With prescription \\
\hline 1 & 7 & 12 \\
\hline 2 & 2 & 10 \\
\hline 3 & 2 & 20 \\
\hline 4 & 0 & 20 \\
\hline 5 & 2 & 5 \\
\hline 6 & 3 & 7 \\
\hline 7 & 3 & 7 \\
\hline 8 & 5 & 25 \\
\hline 9 & 1 & 4 \\
\hline 10 & 1 & 2 \\
\hline 11 & 2 & 4 \\
\hline 12 & 1 & 1 \\
\hline 13 & 6 & 4 \\
\hline 14 & 3 & 4 \\
\hline 15 & 1 & 5 \\
\hline 16 & 0 & 10 \\
\hline
\end{tabular}

\section{Factors perceived by pharmacists to be contributing to antibiotics dispensing without prescription}

Pharmacists felt safe to dispense antibiotics without prescription if the consumers demanded the medication by name. Other factors included lack of confidence on physician diagnosis, consumers' pressure, emergency cases, financial considerations, pharmaceutical companies, pharmacy management, time pressure, and easy access to community pharmacies.

The majority of the pharmacists said that they would dispense antibiotics if the consumers requested the drug by name, assuming that they would be familiar with it and had used it properly before.

"When a patient comes to me and ask for antibiotics I usually ask him a few questions to make sure he is honestly sick, then I give him whatever antibiotics he requested." (Community pharmacist 2)

"I will dispense antibiotics without prescriptions to patients if they requested the drug by name because this means they have used it before and familiar with the use" (Community pharmacist 10)

Lack of confidence on physician diagnosis was perceived as one of the factors that encourage pharmacists to dispense antibiotics: "Physicians diagnose the case better than pharmacists, but even physicians do not ask for all the required laboratory investigations." (Community pharmacist 6)

"If I feel an antibiotic is indicated for the particular patient, I will immediately dispense it, because even physicians do not perform all the necessary laboratory tests and sometimes they prescribe antibiotics without doing any laboratory test." (Community pharmacist 9)

Pressure from the consumers to dispense antibiotics was one of the factors that force the pharmacists to dispense antibiotics.

"First I try to advise the patient about the risk of using antibiotics without prescription, but if they don't listen to me then I will prescribe the antibiotic" (Community pharmacist 1)

Unavailability of clinics after office hours or emergency cases was the reasons given for dispensing antibiotics without prescription.

"Sometimes patients come to me early in the morning at 3 am, and there are no clinics at that time." (Community pharmacist 5)

"If a patient requested antibiotics in emergency case and he doesn't want to visit the physician, I will dispense antibiotics to him." (Community pharmacist 9)

Some pharmacists assumed that they would lose their clients if they refuse to sell antibiotics without prescription.

"Sometimes I will dispense antibiotics to avoid losing my customers" (Community pharmacist 2)

"You should keep one thing in your mind give patient what they need, not what you want them to buy; the most important factor in business success is trust building" (Community pharmacist 10)

Majority of the pharmacists believed that promotions and bonuses from pharmaceutical companies on particular drugs' sales influenced their practice:

"Pharmaceutical companies give us bonus if we sell more antibiotics, but we dispense antibiotics only when needed" (Community pharmacist 16)

The pressure from pharmacy management on pharmacists to reach certain financial targets was commonly given by pharmacists as a reason to promote irrational use of antibiotics.

"Some pharmacy management asks the pharmacist for certain financial targets otherwise they will kick them out so they tend 
to sell more antibiotics because they are too expensive but some pharmacists concentrate on the selling of cosmetics to reach that profit" (Community pharmacist 1)

Consumers prefer to simply go to any community pharmacy and buy antibiotic to save time. This perception has encouraged pharmacists to practice irrational use of antibiotics.

"They want antibiotics and at the same time they do not want to visit the clinics or Hospitals, even if you advise them they always say I do not have time to visit doctor or I do not have money" (Community pharmacist 5)

\section{Factors preventing pharmacists to dispense antibiotic without prescription}

Lack of complete medical history about the patient and patients with complicated symptoms were perceived by pharmacists as discouraging antibiotics dispensing without prescription.

"If I do not know the full patient medical history I will refuse to dispense antibiotic, but I know that he or she is going to buy it from other pharmacies." (Community pharmacist 9)

"If I feel that the case is very serious and complicated I will refuse to dispense antibiotics." (Community pharmacist 10)

\section{Discussion}

The study found that, patients can buy antibiotics from community pharmacies without medical prescription, either by demanding the drug by name or by simply explaining the symptoms to the pharmacists. In many cases pharmacists did not follow the rules and regulations for nonprescription drugs and showed an alarming irresponsible practice that encourages misuse of antibiotics. Pharmacists perceived that requests for specific antibiotics by name and financial considerations were good reasons to freely dispense antibiotics without prescriptions. This could be due to pharmacists' lack of knowledge and absence of strict regulations. However few pharmacists believed that lack of regulations is one of the factors leading to irrational use of antibiotics. It is not surprising that all the pharmacists that were interviewed were non-Saudi because of the shortage of national pharmacists in community pharmacies, as Saudi pharmacists usually prefer to work in the government healthcare settings. Similarly only male pharmacists are allowed to work in the community pharmacies in Saudi Arabia. The study reported that majority of the pharmacists dispensed antibiotics without prescription. Previous studies have also shown similar trends [6,10,15-17]. Community pharmacists have demonstrated a serious lack of knowledge regarding antibiotics as also seen in other developing countries $[15,17]$. They perceived dispensing of antibiotics without prescription to be a safe practice. However, it is difficult for a pharmacist to dispense the right antibiotic for the right patient, because of the difficulties in the differentiation between viral and bacterial infection, unknown medical history and possible adverse outcomes. A study from Egypt, a neighbouring country, reported that $54 \%$ of the antibiotics recommended by community pharmacists were inappropriate [18]. Pharmacists' also mentioned that they could not resist the increasing demand for antibiotics, believing that patients may buy it from other community pharmacies. This could be the influence of cultural beliefs that antibiotics are a cure for all illnesses [19]. Financial interests of pharmacy owners and bonuses from pharmaceutical companies play an important role in this regard, as pharmacists' afraid to lose their customers [20]. In India a study reported that financial interests of community pharmacies played an important role in irrational use of antibiotics [17]. Few pharmacists' mentioned that they would not dispense antibiotic in severe cases and for patients with incomplete or suspicious medical history. This was possibly to protect themselves from any negative consequences that could make the pharmacist legally accountable. One of the study's limitations might be that the interviews were conducted during pharmacists working hours and therefore the pharmacists might have been interrupted by customers several times during the interviews. This may have led to distraction and loss of focus during the interviews. The reliability of the data was maintained by coding the transcript by two different researchers. To maintain the credibility of the data the researchers self-interpretations and emotion, sentiments and contemplation were avoided. This is the first study exploring community pharmacists view about antibiotics dispensing without prescriptions in Saudi Arabia.

In conclusion pharmacists reported that antibiotics were frequently dispensed without prescription. This finding strongly urges the need to change community pharmacists practice towards antibiotics misuse. It is noteworthy to mention that antibiotic misuse in Saudi Arabia has not changed over the last two decades $[21,22]$. It is the responsibility of healthcare authorities in Saudi Arabia to educate pharmacists and strengthen the rules and regulations which keep the rights of consumers while protecting them from antibiotic misuse and rule violation. On the other hand, consumers should be educated about the risk of seeking antibiotics without prescription through public campaigns and media to increase their awareness about prescription and nonprescription medications. Particularly, consumers should be educated about seeking treatment for minor illness from primary care centers and not community pharmacies.

\section{Conclusion}

In conclusion, pharmacists have shown an alarming irresponsible practice that encourages public misuse of antibiotics. Several factors encouraged pharmacists to dispense antibiotics without prescriptions including economic consideration and increasing demand by the public.

\section{Recommendation}

Antibiotics misuse by the public is very common especially in developing countries. Pharmacists should not dispense antibiotics without prescription to the public; instead they should advice patients to seek physician advice. The ministry of health in Saudi Arabia has recently imposed sanctions on pharmacists who do not comply with rational antibiotics 
study

dispensing. This role has increased public knowledge about the risk of antibiotics misuse. Further studies are needed to evaluate the effectiveness of the new sanctions on rational use and dispensing of antibiotics.

\section{Acknowledgment}

The authors would like to thank the participants for accepting to participate in this study.

\section{References}

1. Bin Abdulhak AA, Altannir MA, Almansor MA, Almohaya MS, Onazi AS, Marei MA, Aldossary OF, Obeidat SA, Obeidat MA, Riaz MS, Tleyjeh IM. Nonprescribed sale of antibiotics in Riyadh, Saudi Arabia: a cross sectional study. BMC Public Health 2011; 11: 538.

2. Aljadhey H, Mahmoud MA, Hassali MA, Alrasheedy A, Alahmad A, Saleem F, Sheikh A, Murray M, Bates DW. Challenges to and the future of medication safety in Saudi Arabia: a qualitative study. Saudi Pharm J 2014; 22: 326-332.

3. Okeke IN, Lamikanra A, Edelman R. Socioeconomic and behavioral factors leading to acquired bacterial resistance to antibiotics in developing countries. Emerg Infect Dis 1999; 5: 18-27.

4. Wright GD. Q\&A: antibiotic resistance: what more do we know and what more can we do? BMC Biol 2013; 11: 51.

5. Antimicrobial Global Report on Surveillance 2014. World Health Organiazation.

6. Farah R, Lahoud N, Salameh P, Saleh N. Antibiotic dispensation by Lebanese pharmacists: a comparison of higher and lower socio-economic levels. J Infect Public Health 2015; 8: 37-46.

7. Dameh M, Green J, Norris P. Over-the-counter sales of antibiotics from community pharmacies in Abu Dhabi. Pharm World Sci 2010; 32: 643-650.

8. Mukonzo JK, Namuwenge PM, Okure G, Mwesige B, Namusisi OK, Mukanga D. Over-the-counter suboptimal dispensing of antibiotics in Uganda. J Multidiscip Healthc 2013; 6: 303-310.

9. Hounsa A, De Mol P. Antibiotics dispensed upon the recommendation of staff in private dispensaries in Abidjan in the Ivory Coast. J Pharm Belg 2009; 99-104.

10. Llor C, Cots JM. The sale of antibiotics without prescription in pharmacies in Catalonia, Spain. Clin Infect Dis 2009; 48: 1345-1349.
11. Vaananen MH, Pietila K, Airaksinen M. Self-medication with antibiotics-does it really happen in Europe? Health Policy 2006; 77: 166-171.

12. Patton M. Qualitative evaluation and research methods (3rd Edn.). Beverly Hills, CA 2002

13. Berg BL. Qualitative research methods for the social sciences (6th Edn.). Boston: Allyn \& Bacon 2007.

14. Todd NJ, Jones SH, Lobban FA. Recovery in bipolar disorder: how can service users be supported through a self-management intervention? A qualitative focus group study. J Ment Health 2012; 21: 114-126.

15. Wachter DA, Joshi MP, Rimal B. Antibiotic dispensing by drug retailers in Kathmandu, Nepal. Trop Med Int Health 1999; 4: 782-788.

16. Volpato DE, de Souza BV, Dalla Rosa LG, Melo LH, Daudt CA, Deboni L. Use of antibiotics without medical prescription. Braz J Infect Dis 2005; 9: 288-291.

17. Kotwani A, Wattal C, Joshi PC, Holloway K. Irrational use of antibiotics and role of the pharmacist: an insight from a qualitative study in New Delhi, India. J Clin Pharm Ther 2012; 37: 308-312.

18. Sabry NA, Farid SF, Dawoud DM. Antibiotic dispensing in Egyptian community pharmacies: an observational study. Res Social Adm Pharm 2014; 10: 168-184.

19. Okumura J, Wakai S, Umenai T. Drug utilisation and selfmedication in rural communities in Vietnam. Soc Sci Med 2002; 54: 1875-1886.

20. Igun UA. Reported and actual prescription of oral rehydration therapy for childhood diarrhoeas by retail pharmacists in Nigeria. Soc Sci Med1994; 39; 797-806.

21. Bawazir S. Prescribing pattern at community pharmacies in Saudi Arabia. Inter Pharm J 1992; 6: 222-224.

22. Aljadhey H, Assiri GA, Mahmoud MA, Al-Aqeel S, Murray M. Self-medication in Central Saudi Arabia. Community pharmacy consumers perspectives. Saudi Med J 2015; 36: 328-334.

\section{*Correspondence to}

Mansour A Mahmoud

Clinical and Hospital Pharmacy Department

College of Pharmacy

Taibah University

Saudi Arabia 